\title{
Modelo de contrato de terceirização de manutenção: uma abordagem principal-agente
}

\author{
Jonas Alves de Paiva ${ }^{a *}$, Fernando Menezes Campello de Souza ${ }^{\mathrm{b}}$ \\ a*jonas@ ct.ufpb.br, UFPB, Brasil \\ bfmcs@hotlink.com.br, UFPE, Brasil
}

\begin{abstract}
Resumo
A terceirização é uma das soluções utilizadas para se reduzir esforços em atividades não relacionadas com a atividade de produção. Na terceirização da manutenção, modelos de contratos de incentivos existentes na literatura focam como principais indicadores de controle apenas o tempo de reparo e o custo das atividades de manutenção. 0 principal objetivo desse trabalho consiste em apresentar um modelo de incentivos considerando também outras variáveis que são afetadas pela manutenção e que afetam fortemente o lucro, que são a qualidade dos produtos produzidos e a redução da capacidade de produção. 0 trabalho utiliza a teoria principal-agente para modelar um contrato de incentivos que conduz a um aumento do lucro da empresa, forçando o agente a desenvolver atividades que maximizem esse lucro. É apresentada uma exemplificação numérica, que evidencia o impacto positivo nos resultados da empresa, além da generalização e adequação do modelo.
\end{abstract}

Palavras-chave

Contratos de manutenção. Qualidade da manutenção. Teoria principal-agente.

\section{Introdução}

Um ambiente de crescimento econômico e de alta competitividade conduz os gestores, em seus processos de tomadas de decisão, a conhecer e analisar os processos produtivos e os respectivos custos associados a eles, visando minimizar os impactos financeiros gerados pelas atividades empresariais. A fim de garantir a sobrevivência, exige-se também uma visão de mercado e planejamento estratégico do negócio, que pode não ser atingida ao se concentrar esforços naquelas atividades ditas periféricas, tais como segurança, alimentação, treinamento e manutenção. A terceirização, segundo Giosa (1999), é um processo de gestão em que se decide repassar algumas atividades para terceiros, e com os quais deve-se estabelecer relações de parceria, devendo a empresa focar nas demais atividades de produção propriamente ditas. Nessa perspectiva, muitas empresas recorreram ultimamente à prática da terceirização como alternativa para se aumentar a eficiência dos seus processos, haja vista que, ao terceirizar atividades, a empresa se concentra nas atividades fins de seu produto, ou seja, naquilo que ela é capaz de fazer melhor, com competitividade e maior produtividade.

As tarefas secundárias e auxiliares são realizadas por empresas que se especializaram de maneira mais racional e com menor custo (FARIA, 1994). Tavares (2004) alerta que essas atividades secundárias retiram o foco das empresas das atividades essenciais por não estarem relacionadas à cadeia de valor do produto final. Com a adoção da terceirização dessas atividades, os gestores retomam sua atenção para os processos core da empresa.

No entanto, é preciso enfatizar que, embora periféricas, atividades como gerenciamento de recursos humanos, treinamento, alimentação, segurança, limpeza e manutenção são imprescindíveis para o funcionamento das organizações e exercem papel fundamental na produtividade de qualquer empresa. No caso da manutenção industrial, enquanto o sistema de produção gera produtos e serviços como resultado do próprio sistema, a manutenção provê horas disponíveis para o sistema. Dessa forma, essa 
atividade tem papel essencial nos resultados dos sistemas e exige do gestor um forte controle sobre ela, sendo terceirizada ou não.

0 propósito da manutenção é produzir confiabilidade da capacidade da planta fabril. Confiabilidade pode ser traduzida, segundo Palmer (1999), como a disponibilidade da planta da fábrica para realizar suas atividades fabris gerando, consequentemente, maior produtividade dos recursos. Logo, os custos totais da companhia serão reduzidos ao se aplicar manutenção efetiva em virtude do aumento dessa disponibilidade dos recursos.

Ao se transferir uma atividade tão importante, a empresa não pode ficar à mercê dos contratos oferecidos pelas empresas de manutenção. Ela mesma deve modelar um sistema de incentivos que vise direcionar os esforços para obtenção de resultados positivos.

A teoria principal-agente apresenta bases conceituais para a elaboração desses contratos, através de uma política de incentivos. 0 fundamento da teoria pauta-se no fato de considerar como principal um indivíduo, ou uma empresa, que precise transferir (terceirizar) a outra pessoa/empresa(agente) a realização de certas atividades ou, até mesmo, a fabricação de produtos. No caso da terceirização da manutenção, o custo da realização das atividades é ponto gerador de conflito entre a empresa contratante (principal) e a contratada (agente). Qual o valor ideal a ser pago às empresas contratadas? 0 agente é ciente de que, ao aprimorar suas atividades de manutenção, a disponibilidade das máquinas será aumentada para o principal, mas esse aprimoramento gera um esforço financeiro adicional. 0 problema do principal é criar um sistema de incentivos que induza o agente a realizar a melhor ação conforme seu ponto de vista (VARIAN, 1992), mas que traga benefícios para ambas as partes. Portanto, um sistema de incentivos é essencial para garantir que as empresas caminhem numa única direção, que é a de maximização do lucro.

Os modelos encontrados na literatura tratam do agente construindo seu contrato, induzindo o principal ao pagamento de um valor de equilíbrio aos dois. Nas novas modalidades de contratos já constam inclusão de variáveis, tais como MTBF (tempo médio entre falhas) e o MTTR (tempo médio de reparo), apresentadas pelo agente (ALMEIDA; SOUZA, 2001). Essa preocupação com o tempo gasto pela manutenção para restabelecer o funcionamento do sistema surge nos atuais modelos desenvolvidos para contratos de terceirização usando a teoria principal-agente.

Ao se analisarem alguns estudos sobre o tema, verifica-se que os modelos de incentivos consideram apenas os esforços na redução do tempo de interrupção da máquina como variável fundamental da política de incentivos. Essa variável é fortemente influenciada pela estrutura de manutenção adotada pelo agente e conhecimento técnico da equipe. Essa visão é fundamentada na ideia do aumento da disponibilidade da planta fabril.

Murthy e Yeung (1995) modelam duas opções de contratos de incentivo buscando a maximização do lucro do agente, considerando a taxa de falhas e o custo da realização da manutenção para o primeiro modelo e os custos por unidade de tempo no segundo modelo. Murthy e Ashgarizadeh (1999) complementam o modelo anterior com uma modelagem para contratos de serviços de manutenção, no qual o tempo de reparo é variável fundamental, expandindo depois o estudo em Ashgarizadeh e Murthy (2000), para o caso em que o agente tem mais de um cliente a atender, afetando assim sua taxa de atendimento. 0 modelo é construído a partir de duas opções de oferta, o tempo de reparo e o custo desse serviço de reparo, considerando os efeitos no tempo de atendimento e no custo do agente em realizar esses reparos. Outra aplicação desses modelos foi feita para mais de um canal de atendimento (MURTHY; ASHGARIZADEH, 1999). Jackson e Pascual (2008), baseado no modelo de Ashgarizadeh e Murthy, expandiram a aplicação para o caso no qual os equipamentos encontram-se no fim da vida útil e, portanto, estão na fase de aumento da sua taxa de falhas, explicitando as vantagens do modelo considerado.

Almeida (2001) modela a seleção de contratos baseados em decisão multicritério, avaliando o tempo de interrupção e o custo da intervenção, combinando-os numa função utilidade multiatributo. Posteriormente, o mesmo autor (ALMEIDA, 2005) modela a decisão baseado em utilidade usando o método ELECTRE 1. Tseng et al. (2009) modelam um contrato considerando a adoção futura de uma nova tecnologia em uma estrutura de um sistema já estabelecido, considerando a estimativa dos tempos de parada totais e os custos atrelados. Yeh, Kao e Chang (2009) modelam incentivos para equipamentos alugados, aplicando uma política de manutenção preventiva ótima considerando custos e tempo de atendimento. Já Tarakci, Tang e Teyarachakul (2009) modelam uma política de incentivos baseada na redução do tempo de realização das atividades e na redução do custo através dos efeitos de aprendizagem ao longo do tempo. Wang (2010), por sua vez, modela a decisão de escolha baseado no valor esperado da disponibilidade, cuja variável de decisão é a taxa de atendimento dos reparos.

Percebe-se, portanto, a partir da leitura dos trabalhos de todos esses autores, que os modelos apresentados são construídos pelo agente considerando, justificadamente, a disponibilidade da planta fabril como principal variável de controle, disponibilidade 
esta que é diretamente relacionada com o tempo de reparo das máquinas. Verificam-se apenas alterações nas modelagens conforme as diversas situações existentes no chão de fábrica. Porém, outras variáveis importantes não podem deixar de ser consideradas na modelagem, pois, podem ser afetadas pelo nível das atividades de manutenção, tais como a qualidade do produto final, através do aumento da produção de produtos não conformes, e possíveis reduções da capacidade de produção do sistema provocadas por queda de desempenho da máquina reparada.

As atividades de manutenção, na tentativa de reduzir os tempos de atendimento, podem incorrer em falhas na qualidade de seus serviços. Por esse motivo, alguns trabalhos relacionam essas falhas de atividade à qualidade dos produtos. Ben-Daya e Duffuaa (1995) discutem que as atividades de manutenção são extremamente importantes, pois afetam o desempenho dos equipamentos e a qualidade do produto final. Nesse trabalho realçam-se os efeitos da manutenção na qualidade do produto final. Marchi, Carvalho e Morais (2001) apresentam um modelo de avaliação de uma política de manutenção, através da análise estatística das peças defeituosas produzidas. Já Oke, Ayomoh e Oyedokun (2007) apresentam uma abordagem de medida da qualidade de performance das atividades de manutenção, através da análise do número de unidades produzidas defeituosas ou que requerem retrabalho oriundas de mau funcionamento do sistema de produção. Alguns outros trabalhos tratam da mesma temática, como os de Kumar, Markeset e Kumar (2004) e Stremersch, Wuyts e Frambach (2001), mas sem modelar essas variáveis para construção de contratos.

Portanto, para se modelar contratos de incentivo para serviços de manutenção devem ser considerados os diversos efeitos dessas atividades na produtividade da empresa, além da disponibilidade já considerada nos modelos vigentes na literatura. Identificar meios de mensurar e controlar essas outras variáveis nos modelos é fundamental para se direcionar as ações do agente. 0 modelo que foi desenvolvido neste trabalho agrega como medidas de desempenho das atividades, além da disponibilidade como variável fundamental na determinação da qualidade da manutenção, um indice de qualidade da manutenção relacionado com a produção de peças não conformes e a medida da capacidade de produção do sistema de produção, o que servirá se base para aperfeiçoamento dos modelos de incentivos futuros.

\section{Conceitos importantes para construção do modelo}

Com o intuito de se iniciar o desenvolvimento do modelo é essencial entender alguns conceitos fundamentais que venham a esclarecer os aspectos considerados na sua construção. Inicia-se com a teoria principal-agente, que é base conceitual da modelagem de incentivos.

\subsection{Teoria principal-agente}

A teoria principal-agente é utilizada na modelagem de incentivos em situações em que uma pessoa (o principal) quer delegar a execução de uma atividade para outra pessoa (o agente). Na maioria dos casos, o principal terá dificuldades em notar o esforço aplicado pelo agente, mas deverá observar alguma saída da atividade que seja resultado direto do esforço aplicado na sua execução. 0 problema do principal é designar, no contrato, uma "forma de pagamento" que incentive o agente a agir da melhor forma possível do ponto de vista do principal (MAS-COLELL; WHINSTON; GREEN, 1995).

0 relacionamento entre a empresa contratante e a empresa contratada pode ser tão complexo quanto o relacionamento entre dois seres humanos (MARCHALEK; REBELATO; RODRIGUES, 2007). Jensen e Meckling (1976) ressaltam que quando dois indivíduos buscam maximização de suas utilidades, então existe uma boa razão para acreditar que os agentes não agirão na defesa do interesse do principal. Eles apresentam os mecanismos de construção de modelos de incentivos baseados nos custos de pagamento e de esforços do principal e agente respectivamente.

Os modelos matemáticos que surgem na literatura da relação entre as partes envolvidas no contrato consideram dois problemas centrais: desconhecimento da competência do agente para execução da atividade (informação privada) e objetivos diferentes entre principal e agente. Os dois tipos de informação privada são apresentados em Laffont e Martimort (2001), ou o principal não consegue observar o esforço do principal - conhecido na literatura como moral hazard ou hidden action - ou o agente tem informação privada sobre seus custos, conhecida como adverse selection ou hidden knowledge.

Recomenda-se a leitura de Mas-Collel, Whinston e Green (1995), Varian (1992), Jensen e Meckling (1976) e Laffont e Martimort (2001). Apresentam-se abaixo as equações do modelo básico retirado de Laffont e Martimort (2001), para contratos ótimos de informação completa.

A equação de ganho do principal é dada por:

$W p=R\left(q^{*}\right)-t\left(q^{*}\right)+F$

Sendo:

- Wp - o resultado do lucro obtido com a delegação da atividade; 
- $R\left(q^{*}\right)$ - as receitas ou ganhos obtidos, que é função do resultado (q) da atividade;

- $\mathrm{t}\left(\mathrm{q}^{*}\right)$ - representa o valor pago pela obtenção do resultado (q);

- F - constante considerada para justificar a delegação da atividade, não sendo considerada nos modelos.

Percebe-se que o principal obterá, como resultado do processo de delegação da atividade, um valor líquido que é a diferença entre o que ele recebeu do resultado menos o que ele paga ao agente para obter esse resultado.

0 principal fica sujeito à restrição de compatibilidade de incentivos dada por:

$R\left(q^{*}\right)-t\left(q^{*}\right) \geq 0$

0 principal terá que ter como resultado um lucro positivo.

Já o agente fica sujeito às restrições de participação, sendo dadas por:

$t(q)-\theta q \geq 0$

A restrição é válida independente de o agente ser eficiente ou não eficiente. Portanto, o modelo deve considerar as restrições de compatibilidade de incentivos e de restrição de participação.

\subsection{Terceirização}

Amato Neto (1995), em seu estudo sobre reestruturação industrial, terceirização e redes de subcontratação, apresenta os principais fatores que conduziram as empresas para os processos de desintegração vertical (redução do tamanho da cadeia de atividades de uma empresa), forçando as empresas à contratação de fornecedores competentes. Cabe aqui entender que o fato de um fornecedor ser competente não exclui a divergência de interesses, mas que a qualidade de seus serviços proverá certa segurança à empresa contratante. Amadeo et al. (1996) afirmam que a queda do nível de emprego na indústria resultou em um aumento significativo nas atividades de serviços terceirizados especializados, denotando que a terceirização é um caminho sem volta, sendo cada vez mais crescente o volume de empresas que recorrem a empresas especializadas para cuidar das atividades secundárias à produção. A terceirização de atividades produtivas é uma alternativa de gestão legítima nessa busca por melhores resultados empresariais (VALOIS; ALMEIDA, 2009).

Uma parte dos gestores reforça a necessidade da terceirização da manutenção às empresas especializadas, face à importância das atividades de manutenção em função dos seguintes pontos: a) 0 aumento da tecnologia utilizada nas máquinas e equipamentos fabris força as empresas a terem necessidade de mão de obra qualificada, com investimentos maciços em treinamento e capacitação, além de ferramental adequado para este fim;

b) 0 aumento do uso de novas filosofias de gestão da produção, que visam reduzir estoques de produtos acabados e de material em processamento, leva a empresa a sentir mais fortemente o impacto das decisões da gestão da manutenção, já que a capacidade de produção, a qualidade dos produtos e o tempo de parada para manutenção podem representar a parada de toda a planta fabril; e

c) Ao se contratar empresa especializada em manutenção, os custos totais de manutenção tendem a cair devido aos melhores resultados obtidos, fruto do nível de especialização técnica da empresa contratada.

Apenas o fato da importância dos serviços de manutenção não é fator suficiente para que a empresa contratada realize suas atividades da melhor forma possível, pois assim como o principal ela também visa aumentar sua lucratividade e tenderá a reduzir ao máximo os custos com as atividades de manutenção.

A questão da terceirização dos serviços de manutenção e do conflito de interesses entre principal e agente reforça a importância da construção de um modelo usando os conceitos da teoria do principal-agente para modelar contratos que venham a reduzir o conflito de interesses entre as partes, visando induzir uma cooperação. 0 uso de incentivos contratuais, que deixem as empresas com um mesmo foco de aumentar a lucratividade, é meta fundamental de uma gestão de manutenção atual.

\subsection{Disponibilidade (D)}

A disponibilidade é resultado da confiabilidade do sistema de produção e do tempo de reparo gasto para restabelecer o funcionamento ao sistema. A disponibilidade de uma máquina é função da taxa de falhas $\lambda$, e da taxa média de atendimento $\mu$, e representa o tempo disponível da máquina para efetuar as atividades de produção, sendo expressa pela equação abaixo. $M T B F$ representa o tempo médio entre falhas $\left(M T B=\lambda^{-1}\right)$, e $M T T R$ representa o tempo médio para execução de um reparo $\left(M T T R=\mu^{-1}\right)$.

$$
D=\frac{M T B F}{M T B F+M T T R}=\frac{\lambda^{-1}}{\lambda^{-1}+\mu^{-1}}
$$

Adota-se, no modelo, a hipótese de que a taxa de falhas $\lambda$ é constante (período de vida útil física da máquina). Portanto, as falhas acontecem de forma aleatória, sendo a disponibilidade função apenas da taxa de reparo $\mu$. 0 tempo médio de reparo é o tempo gasto pela equipe de manutenção para restabelecer o equipamento à condição de produção. 0 tempo de 
reparo é diretamente ligado à estrutura de manutenção utilizada pela empresa contratada, quanto maior a estrutura, menor será o tempo de reparo e, portanto, maior a disponibilidade.

Apenas a análise do tempo de reparo não determina a qualidade dos serviços de manutenção, pois a máquina pode retornar ao funcionamento em condições de produção inferiores ao momento da parada. É sobre essa condição de funcionamento da máquina, após os reparos, que o modelo desenvolvido nesse trabalho se concentra. Essa condição é representada através da classificação das atividades de manutenção descritas por Pham (2003), que classifica as atividades de manutenção desde a manutenção perfeita, cuja ação restaura o equipamento a sua condição de novo, até a manutenção ruim, quando a ação retorna o equipamento a uma condição pior à anterior ao problema verificado. Higgins e Mobley (2001) apresentam a manutenção como uma arte, porque problemas aparentemente idênticos necessitam e recebem uma variação significativa de abordagens e ações. Sellitto (2005) analisa a formulação de estratégias de manutenção baseadas na confiabilidade e mantenabilidade. Os resultados possíveis das atividades de reparo devem ser considerados e modelados na política de incentivos.

\section{4. Índice de qualidade da manutenção} (IQm)

Serviços de manutenção executados de forma inadequada podem resultar na produção de peças não conformes, fazendo com que a disponibilidade da máquina não se traduza em peças prontas para venda. Segundo Ben-Daya e Duffuaa (1995), melhorar a qualidade do serviço de manutenção significa promover a redução de desperdícios relacionados à produção de peças defeituosas e realização de retrabalhos.

Alguns procedimentos na execução das atividades de manutenção, tais como usar peças de reposição não recomendadas pelo fabricante, executar serviços com mão de obra não qualificada, eliminar dispositivos de controle ou de segurança da máquina visando a redução de custos ou redução do tempo de atendimento podem gerar problemas ao desempenho do sistema. Esses aspectos podem gerar alterações nos parâmetros de funcionamento das máquinas, tais como temperatura, pressão, velocidade. Essas alterações podem contribuir para o surgimento de peças não conformes. Será considerado nesse trabalho o índice de qualidade da manutenção apresentado por Oke, Ayomoh e Oyedokun (2007).

$$
I Q m=\frac{N c}{N t}=1-\frac{N r}{N t}
$$

Sendo $N t$ o número de peças total produzidas em um intervalo de tempo, $\mathrm{Nc}$ e $\mathrm{Nr}$ o número de peças conformes e não conformes produzidas respectivamente nesse mesmo intervalo de tempo.

De posse da equação, observa-se que $0 \leq 1 Q m \leq 1$. $1 Q m=0$ significa que apenas peças não conformes são produzidas, $l Q m=1$ apenas peças conformes são produzidas. Analisa-se, então, que disponibilidade não significa diretamente resultado financeiro, pois peças produzidas podem não se transformar em peças conformes para vendas. Mas, independente de sua venda, uma peça não conforme gera custos, pois é resultado de um esforço de produção. Os modelos que consideram apenas a disponibilidade podem conduzir o agente a se concentrar na velocidade das atividades de manutenção, não se preocupando com os possíveis efeitos da qualidade do produto final. Outra variável que pode ser afetada pelas atividades de manutenção é a redução da capacidade de produção.

\subsection{Capacidade de produção}

A capacidade é o volume de peças que uma unidade produtiva pode produzir na unidade de tempo, afetando diretamente a produtividade. Os pontos citados no tópico anterior, tais como usar peças de reposição não recomendadas pelo fabricante, executar serviços com mão de obra não qualificada, eliminar dispositivos de controle ou de segurança da máquina, além de gerar redução da qualidade dos produtos, podem alterar parâmetros das máquinas que reduzam sua velocidade de produção. Nesse caso, algumas ações de manutenção podem retornar a máquina a uma condição de funcionamento afastado dos padrões de projeto em termos de capacidade.

0 propósito de um planejamento de manutenção deve estar focado em uma alta produtividade, através da aplicação de planejamento e princípios de agendamentos de intervenções. 0 nível de serviço de manutenção, representado por atividades executadas com pessoal qualificado, peças de reposição de boa qualidade e uso de ferramental adequado, reduz consideravelmente as horas de reparo e os custos com manutenção corretiva, além de manter a capacidade de produção do sistema.

Devido a sua relação direta com os resultados da empresa, a capacidade horária de produção das máquinas deve ser considerada pelo principal como variável de controle do agente.

\section{Desenvolvimento do modelo}

\subsection{Notação utilizada}

- $R=$ Receita recebida pelo principal; 
- $P=$ Preço de uma unidade conforme vendida no mercado;

- $W p, W a=$ Lucros financeiros brutos obtido pelo principal e pelo agente respectivamente;

- $C T=$ Custo total do principal;

- $C M p=$ É o atual custo de manutenção do principal antes da terceirização, conforme o seu padrão de produção atual;

- $C M a g=$ É o esforço financeiro que o agente realiza para manter um determinado padrão de manutenção;

- $C V u=$ Custo variável de uma unidade produzida;

- $C F=$ Custo fixo atual da empresa sem terceirização;

- $C F_{-C M p}=$ Custo fixo do principal após a terceirização, sem os custos de manutenção;

- $1 Q m$ = Índice de qualidade da manutenção relacionada às peças conformes;

- $N t=$ Número de peças total produzidas em um intervalo de tempo;

- $N c=$ Número de peças conformes produzidas em um intervalo de tempo;

- $N r=$ Número de peças não conformes produzidas em um intervalo de tempo;

- $D=$ Disponibilidade do sistema de produção;

- $C$ = Capacidade de produção do sistema em um intervalo de tempo;

- $I p=$ Variável de incentivo.

\subsection{Hipóteses do modelo}

As hipóteses consideradas no desenvolvimento do modelo são descritas abaixo:

1 As quantidades produzidas são totalmente vendidas, não havendo limitações de demanda nem alterações dos preços praticados no mercado;

2 A empresa contratante e a contratada conhecem o atual estado de disponibilidade da produção, seu índice de peças defeituosas e a capacidade de produção do sistema. É um contrato baseado em sistema de informação completa;

3 Os desvios da capacidade de produção são devido às atividades de manutenção, sendo desconsiderados os erros oriundos de problemas com mão de obra ou matérias-primas;

4 As peças defeituosas produzidas são devido a problemas de mau funcionamento da máquina, não havendo geração de não conformidades em outras atividades, tais como transporte;

5 A manutenção contratada será completa, sendo de responsabilidade do contratado a compra e a troca de peças de reposição;

6 A máquina está no período de sua vida útil física, sendo a taxa de falhas $(\lambda)$ constante, ou seja, as falhas ocorrem aleatoriamente e o contrato é de longo prazo; e

7 A manutenção contratada será aplicada a uma única máquina (que nesse caso será o sistema) que produz todas as atividades de um único produto.

\subsection{Impacto no lucro bruto do principal}

Para iniciar o entendimento das variáveis consideradas no modelo e as relações possíveis, inicia-se abaixo a estruturação matemática da relação entre estas. Calculando a receita obtida com a máquina:

A receita é dada por:

$R=P . N c$

Sendo,

$N c=D .1 Q m . C$

0 número de peças conformes $N c$ é resultado da disponibilidade $D$ do sistema, do índice de qualidade da manutenção $1 Q m$, que informa o percentual de peças conformes geradas, e da capacidade de produção $C$.

Substituindo a Equação 7 em 6 tem-se:

$R=P \cdot D \cdot 1 Q m \cdot C$

A equação de custos da empresa é dada por:

$C T=C F+C V=C F+C V u \cdot N t$

0 número de peças totais produzidas é resultado da disponibilidade e da capacidade de produção, e tem-se:

$C T=C F+C V u \cdot C \cdot D$

0 lucro bruto, receitas menos os custos, é dado por:

$W p=R-C T=P . D . I Q m . C-C V u . C . D-C F$

$W p=D . C .(P . I Q m-C V u)-C F$

Observando a equação de lucro bruto da empresa, percebe-se que um aumento na disponibilidade e na capacidade de produção resulta em aumento de receita e do custo variável total, mas o custo unitário total ficará menor, devido ao rateio do custo fixo. Já ao observar o $1 Q m$ na Equação 11, vê-se que uma alteração nesse indicador provoca um aumento na receita, mas não gera aumento dos custos da empresa. Verifica-se, portanto, a importância da consideração dessa variável nos contratos.

Apresenta-se na Tabela 1 o cálculo das variações no lucro bruto $(W p)$ provocadas pelas alterações nas variáveis consideradas.

\subsection{Exemplo numérico}

Exemplificando numericamente os impactos das alterações nos valores das variáveis sobre o lucro, 
Tabela 1. Impacto no lucro gerado pelas variáveis consideradas.

\begin{tabular}{ccccccccccccc}
\hline \multicolumn{10}{c}{ Cálculo do lucro bruto } \\
\hline $\mathrm{P}$ & $\mathrm{C}(\mathrm{pcc/h})$ & $\mathrm{D}$ & $\mathrm{Nr}$ & $\mathrm{Nt}$ & $\mathbf{1 Q m}$ & $\Delta \mathrm{lQm}$ & $\mathrm{CVu}$ & $\mathrm{CFm}$ & $\mathrm{CT}$ & $\mathrm{R}$ & $\mathrm{Wp}$ & $\Delta \mathrm{Wp}$ \\
\hline 80 & 150 & 0,8 & 52 & 120 & 0,57 & 0,0 & 20 & 30 & 2430 & 5440 & 3010 & 0 \\
80 & 150 & 0,8 & 48 & 120 & 0,60 & 0,033 & 20 & 30 & 2430 & 5760 & 3330 & 320 \\
80 & 150 & 0,8 & 44 & 120 & 0,63 & 0,033 & 20 & 30 & 2430 & 6080 & 3650 & 320 \\
80 & 150 & 0,82 & 42 & 123 & 0,66 & 0,025 & 20 & 30 & 2490 & 6480 & 3990 & 340 \\
80 & 180 & 0,92 & 32 & 166 & 0,81 & 0,075 & 20 & 30 & 3342 & 10688 & 7346 & 1902 \\
\hline
\end{tabular}

Fonte: modelo desenvolvido pelo autor.

considera-se uma empresa com uma determinada máquina que produz um único produto com capacidade de $C=150 \mathrm{pç} / \mathrm{h}$, gerando desse total 52 peças não conformes. A peça é vendida a $P=80,00$ e a parcela de custo fixo da empresa rateado para a máquina é de $C F m=30,00 /$ hora. 0 custo variável unitário gerado por cada unidade produzida é de $C v u=20,00$. A disponibilidade atual da máquina é de $D=0,8$. 0 lucro bruto $W p$ é calculado conforme Equação 11, e suas variações são obtidas conforme as equações abaixo:

$$
\begin{aligned}
& \frac{\partial W_{p}}{\partial I Q m}=D . C . P ; \frac{\partial W_{p}}{\partial D}=C . P . I Q m-C . C V u ; \\
& \frac{\partial W_{p}}{\partial C}=D . P . I Q m-D . C V u
\end{aligned}
$$

Observando o impacto das variáveis no ganho bruto, conforme Tabela 1, nota-se nas três primeiras linhas que uma redução na produção de 4 peças não conformes $(\mathrm{Nr})$ gera um aumento do lucro de forma constante no valor de $\$ 320,00$. Já nas linhas restantes ocorre a combinação da redução de $N r$, juntamente com o aumento da disponibilidade $D$ e aumento da capacidade $C$, verificando-se que a variação $\Delta W p$ é crescente a cada período. A combinação dessas variáveis deve ser considerada na estruturação dos incentivos, já que há impactos significativos nos resultados da empresa.

\subsection{Sequência do modelo}

Nesta parte do trabalho identifica-se a sequência de como se processa a negociação do contrato conforme a teoria principal-agente:

10 principal considera no contrato as variáveis conhecidas $(D, I Q m, C, C M p, I p)$ e apresenta um contrato ao agente;

20 agente analisa as informações da manutenção, observa seus custos para manter o padrão atual da manutenção, verifica o valor pago e o incentivo fornecido pelo principal, e decide aceitar ou rejeitar o contrato;

3 Se o agente aceitar o contrato, ele recebe uma parcela fixa $C M p$ e uma parcela de incentivos variável $I p$ (variável de incentivo) de acordo com os resultados de melhorias das variáveis $(D, 1 Q m$ e $C)$ estabelecidos; e
4 Caso o agente não aceite o contrato ele fica com sua utilidade reserva, sendo considerada como 0 , que é o seu estado atual sem aceitar o serviço e o principal fica com seu custo de manutenção atual.

\subsection{Equações do modelo}

A equação de lucro bruto do principal é estruturada da seguinte forma:

$$
\begin{aligned}
& W p=D . C .(P . I Q m-C V u)-C F_{-C M p} \\
& -C M p\left(D_{0}, I Q m_{0}, C_{0}\right)-I p .(\Delta D+\Delta I Q m+\Delta C)+\delta
\end{aligned}
$$

A primeira parcela representa o lucro obtido com a venda das unidades conformes, excluindo os custos variáveis unitários de produção $C V u$ de todas as unidades produzidas.

Se o contrato for aceito, o custo fixo da empresa fica reduzido dos custos fixos de manutenção, já que a empresa não estrutura mais a manutenção para esse sistema. Esse novo custo fixo é representado pela segunda parcela da equação.

0 valor da terceira parcela representa o valor fixo que será pago ao agente para executar a manutenção mantendo os padrões atuais $D_{0}, 1 Q m_{o}$ e $C_{0}$, por isso $C M p$ é função do estado inicial da máquina. 0 valor $C M p$ é igual ao valor atualmente gasto na manutenção pelo principal sem terceirização.

0 incentivo $I p$ é a variável de decisão do principal, e será calculado em função das variações na disponibilidade $D$, do índice de qualidade da manutenção $1 Q m$ e da capacidade de produção $C$. Se por acaso as variações forem negativas, o agente será penalizado, pois a parcela de incentivos fica positiva significando uma penalização de receita. Caso o agente não efetue melhorias nas variáveis, este receberá o valor fixo $C M p$ pela manutenção do estado atual.

A parcela $\delta$ representa um ganho adicional obtido por redução de gastos administrativos do principal relacionados às atividades de manutenção, mas que deixarão de existir se o agente aceitar o contrato. $0 \delta$ foi apresentado na função de lucro do principal apenas para alertar sobre esse aspecto, mas ele não será considerado no restante do trabalho.

0 principal estará sujeito à restrição de compatibilidade de incentivos: 
S.a.

$$
\begin{aligned}
& \Delta R-\Delta C p-I p \cdot(\Delta D+\Delta I Q m+\Delta C) \geq 0 \\
& \Delta D=D_{n}-D_{0} ; 1 Q m=\Delta I Q m_{n}-1 Q m_{0} ; \Delta C=C_{n}-C_{0}
\end{aligned}
$$

A restrição 13 evidencia que, caso o agente promova uma variação positiva nas variáveis $(D, C$ ou $1 Q m$ ), ele receberá um valor de incentivo que deverá ser inferior ao lucro $(\Delta R-\Delta C p)$ obtido pelo principal como resultado do aumento do valor dessas variáveis.

As Equações 14 são apresentadas para esclarecer que os valores de incentivos pagos obtidos com a melhoria das variáveis de controle estão relacionados à data " $O$ " de fechamento do contrato, já que o esforço para aumentar o valor de uma das variáveis gerará lucro durante os períodos subsequentes para o contratante. Se os lucros fossem considerados entre períodos, seria mais custoso a cada periodo provocar variações positivas dessas variáveis, chegaria um momento em que o incentivo pago seria 0 , mesmo com um trabalho contínuo de aperfeiçoamento das atividades executadas.

A equação de lucro do agente fica:

$$
\begin{aligned}
W a=C M p & \left(D_{0}, l Q m_{0}, C_{0}\right)-C M a g\left(D_{0}, I Q m_{0}, C_{0}\right) \\
& +I p .(\Delta D+\Delta l Q m+\Delta C)-C M a g(\Delta D+\Delta l Q m+\Delta C)
\end{aligned}
$$

E suas restrições de participação são dadas por: s.a.

$$
\begin{aligned}
& C M p\left(D_{0}, \operatorname{lQm}_{0}, C_{0}\right)-C M a g\left(D_{0}, \operatorname{lQm}_{0}, C_{0}\right) \geq 0 \\
& \operatorname{lp} \cdot(\Delta D+\Delta I Q m+\Delta C)-C \operatorname{Mag}(\Delta D, \Delta I Q m, \Delta C) \geq 0
\end{aligned}
$$

A primeira parcela da Equação 15 representa o valor fixo pago pelo principal para o agente manter os níveis atuais da manutenção. A segunda parcela representa o esforço em termos de custo do agente para manter os níveis atuais de manutenção. Quanto mais eficiente for o agente, menor será esse esforço e, consequentemente, maior será o seu lucro bruto. 0 agente não aceitará o contrato se o seu custo para execução das atividades for inferior ao valor recebido, representado pela equação de restrição 16.

A terceira parcela da Equação 15 representa os incentivos recebidos do principal, caso haja variações positivas nas variáveis consideradas. Se as variações forem negativas, então o agente será penalizado. Para aumentar o valor dessas variáveis é necessária a realização de um esforço (custo) adicional, representado pela quarta parcela da mesma equação. Portanto, o agente será conduzido a melhorar os valores atuais dessas variáveis se o valor de incentivos recebido for superior ao esforço necessário, que é considerada na equação de restrição de participação 17.

Se melhorias nas atividades de manutenção atuais não gerarem aumentos da capacidade de produção e da qualidade dos produtos do sistema, o incentivo fica relacionado apenas à observação da disponibilidade, recaindo sobre os modelos já existentes na literatura. Caso haja possibilidades de melhorias da capacidade e da qualidade dos produtos finais, o agente irá observar seus custos e verificar em qual das variáveis os lucros apresentam melhores resultados, se no aumento da disponibilidade, na redução do número de peças não conformes ou no aumento da capacidade da produção. Verifica-se, portanto, que o agente poderá executar um trade-off entre variações positivas e negativas das variáveis consideradas.

Um exemplo de estrutura da função custo do agente (CMag), para agir melhorando as variáveis, pode ser dada por:

$$
\begin{aligned}
C M a g= & C M a g\left(D_{0}, \operatorname{lQm} m_{0}, C_{0}\right)+K_{1} \sum_{i=1}^{n} C M a g\left(D_{i}-D_{i-1}\right) \\
& +K_{2} \sum_{i=1}^{n} C \operatorname{Mag}\left(\operatorname{lQm}_{i}-\operatorname{lQm}_{i-1}\right)+K_{3} \sum_{i=1}^{n} C \operatorname{Mag}\left(C_{i}-C_{i-1}\right)
\end{aligned}
$$

A primeira parcela representa o custo gasto pelo agente para manter os níveis atuais de manutenção. Para agir, no sentido melhorar a manutenção, será necessário realizar um valor específico de esforço $C M a g(\Delta x)$, que é estabelecido pela natureza do sistema e não pelo agente. 0 que determina o valor do custo total do agente serão os $K s$, que estão diretamente relacionados com a sua eficiência. $\mathrm{Na}$ equação de custo, os Ks estão separados, pois a eficiência poderá ser diferente para realização de melhorias em cada uma das variáveis consideradas.

As parcelas 2, 3 e 4 representam o custo gerado para se sair do estado inicial e se chegar ao estado $n$. Caso não haja variação, as parcelas de custo serão nulas.

O modelo fica aberto para a utilização de diversas possibilidades de funções custos, oriundas das diferentes naturezas dos sistemas de produção.

\section{Resultados}

Uma das formas de modelar o contrato satisfazendo as restrições apresentadas, e que promova cooperação do agente devido ao incentivo pago $l p$, será considerar, nesse caso, que o principal e o agente obtêm o mesmo aumento no lucro, fruto das atividades de melhoria. Portanto, as equações de variação no lucro total serão igualadas. Essa solução é dada por se considerar um modelo de informação completa.

\subsection{Determinação da equação do incentivo (Ip)}

Como a parcela $C M P(D o, 1 Q m o, C o)$ paga pelo principal é igual ao seu custo atual de manutenção, considera-se o custo fixo com essa parcela. Chamando $W p_{\text {-lp }}$ de lucro inicial do principal sem considerar os valores de manutenção e incentivos pagos ao agente, tem-se:

$$
\text { D.C. }(P .1 Q m-C V u)-C F=W p_{-I p}
$$


Obtém-se da primeira restrição do agente da Equação 16:

$$
\operatorname{CMp}\left(D_{0}, l Q m_{0}, C_{0}\right)=\operatorname{CMag}\left(D_{0}, l Q m_{0}, C_{0}\right)
$$

lgualando os ganhos das funções de lucro:

$$
\Delta W p=\Delta W a
$$

Tem-se, portanto:

$$
\begin{aligned}
& \Delta W p=W p_{f-i p}-l^{*} p .(\Delta D+\Delta l Q m+\Delta C)-W p_{0-l p} \\
& \Delta W a=i p .(\Delta D+\Delta l Q m+\Delta C)-C M a g\left(D_{0}, I Q m_{0}, C_{0}\right) \\
& -C M a g(\Delta D, \Delta l Q, \Delta C)+C M a g\left(D_{0}, I Q m_{0}, C_{0}\right)
\end{aligned}
$$

$\mathrm{Na}$ Equação 22, o lucro final do principal será reduzido do incentivo pago para se obter a melhoria e do valor inicial do lucro sem melhorias. Na Equação 23 do agente, verifica-se que o novo lucro do agente é dado pelo valor de incentivo recebido para promover melhorias, menos o seu custo de manutenção para manter o estado atual, menos o seu esforço para promover essa melhoria. A parcela final é o custo de se manter o estado atual da manutenção, ficando positivo porque a equação representa a variação no lucro.

Igualando as Equações 22 e 23, substituindo a equação 19 na Equação 22 e isolando o valor do incentivo, tem-se:

$\dot{l p}=\left(\frac{P\left(D_{f} l Q m_{f} C_{f}-D_{0} l Q m_{0} C_{0}\right)-C V_{u}\left(D_{f} C_{f}-D_{0} C_{0}\right)+C M a g(\Delta D, \Delta l Q m, \Delta C)}{2 .(\Delta D+\Delta l Q m+\Delta C)}\right)(24)$

\section{$\forall \Delta D, \Delta l Q m$ ou $\Delta C \neq 0$}

Analisando-se a Equação 24, vê-se que o incentivo leva em consideração o custo de manutenção para se gerar as variações. Esse custo será retirado do lucro adicional obtido no sistema, pois a parcela positiva provoca um aumento no incentivo $I p$ reduzindo o lucro do principal conforme o custo de manutenção.

Para o principal, de acordo com a Equação de restrição 13, ele analisa que:

$$
\Delta R-\Delta C T \geq \operatorname{lp} .(\Delta D+\Delta / Q m+\Delta C)
$$

0 principal verificará que a variação no seu lucro deve ser maior que o valor de incentivo pago.

Para o agente, de acordo com a restrição 2, Equação 17, reescrevendo tem-se:

$$
\begin{aligned}
& K_{1} \cdot \sum_{i=1}^{n} C M a g\left(D_{i}-d_{i-1}\right)+K 2 \cdot \sum_{l=l}^{n} C M a g\left(I Q m_{i}-l Q m_{i-1}\right) \\
& +K 3 \sum_{i=1}^{n} C \operatorname{Mag}\left(C_{i}-C_{i-1}\right) \leq I p \cdot(\Delta D+\Delta l Q m+\Delta C)
\end{aligned}
$$

0 agente observará suas equações de custo individualmente, analisando em qual das variáveis ele irá atuar para maximizar seus resultados. Caso haja situações onde o aumento não seja mais possível, essa parcela será nula.
Para melhor entendimento, apresenta-se um caso em que apenas possam ser executadas melhorias no número de peças conforme; as Equações 24 e 26 tornam-se:

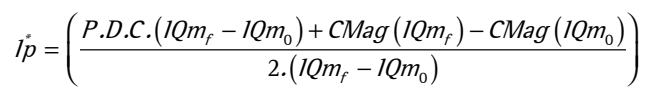

e

$$
K 2 \cdot \sum_{i=1}^{n} C M a g\left(1 Q m_{i}-1 Q m_{i-1}\right) \leq l p
$$

As demais parcelas foram zeradas e desconsideradas, já que não há possibilidade de melhorias dessas variáveis.

\subsection{Exemplo numérico}

Considerando-se os valores do exemplo da seção 3.4, acrescentando-se o custo de manutenção da empresa com a referida máquina de $C M p=10,00 /$ hora, chegam-se aos dados da Tabela 2.

Percebe-se o impacto no lucro da empresa ao se reduzir o número de peças não conformes $\mathrm{Nr}$ mantendo as demais variáveis constantes. 0 cálculo do lucro bruto apresentado é calculado pela Equação 12 e só pode ser atingido com esforço adicional necessário para promover redução do $N r$. 0 lucro calculado na Tabela 2 não seria atingido, já que a empresa necessitaria de um esforço $C M p$ maior do que os $\$ 10,00$ para aumentar o $1 Q m$ da manutenção.

Será analisado o caso da máquina com as atividades de manutenção terceirizada, considerando que o agente vai trabalhar mantendo o nível de manutenção atual e irá reduzir o número de peças não conformes.

Cada empresa tem sua própria função de custos. No modelo pode-se operar com diversas funções. Considera-se, para demonstrar a flexibilidade do modelo, que a função de custos $C M a g(1 Q m)$ do agente em questão é dada por:

$$
C M a g(I Q m)=K_{2} \cdot e^{\left(\theta^{*} l Q m\right)}
$$

$0 \theta$ define a tecnologia do sistema de produção estudado. Quanto maior a tecnologia, maiores serão os custos necessários de manutenção para manter um padrão de qualidade, e $K_{2}$ representa a eficiência do agente sobre essa tecnologia. A função considerada no modelo é exponencial, que se adequa à maioria dos sistemas, pois considera que o esforço de melhorias requer crescimento exponencial de custos. Considerando os valores da Tabela 2 e acrescentando-se os valores de custo do agente para um $\theta=5$ e um $K_{2}=1,5$ e aplicando-se o modelo, chega-se aos valores da Tabela 3.

Analisando-se os valores obtidos na Tabela 3, verifica-se que o aumento no lucro obtido com a redução das peças não conformes não fica em sua totalidade com o principal, pois ele deve pagar o 
Tabela 2. Impacto no lucro com redução de peças não conformes produzidas.

\begin{tabular}{ccccccccccccc}
\hline \multicolumn{10}{c}{ Sem terceirização } \\
\hline Preço & $\mathrm{C}(\mathrm{pc} / \mathrm{h})$ & $\mathrm{D}$ & $\mathrm{Nr}$ & $\mathrm{Nt}$ & $\mathbf{I Q m}$ & $\Delta \mathrm{lQm}$ & $\mathrm{Cvu}$ & $\mathrm{CF}$ & $\mathrm{CT}$ & $\mathrm{R}$ & $\mathrm{CMp}$ & Lucro (W) \\
\hline 80 & 150 & 0,8 & 52 & 120 & 0,57 & 0,01 & 20 & 30 & 2430 & 5440 & 10 & 3010 \\
80 & 150 & 0,8 & 48 & 120 & 0,60 & 0,03 & 20 & 30 & 2430 & 5760 & 10 & 3330 \\
80 & 150 & 0,8 & 44 & 120 & 0,63 & 0,07 & 20 & 30 & 2430 & 6400 & 10 & 3650 \\
80 & 150 & 0,8 & 42 & 120 & 0,65 & 0,07 & 20 & 30 & 2430 & 7040 & 10 & 3810 \\
\hline
\end{tabular}

Fonte: modelo desenvolvido pelo autor.

Tabela 3. Impacto no lucro com a atividade terceirizada.

\begin{tabular}{|c|c|c|c|c|c|c|c|c|c|c|c|c|}
\hline \multicolumn{13}{|c|}{ Com terceirização } \\
\hline $\mathrm{P}$ & $\mathrm{C}(\mathrm{pc} / \mathrm{h})$ & D & $\mathrm{Nr}$ & $1 Q m$ & $\Delta \mathrm{IQm}$ & CT & $\mathrm{R}$ & K2 & $\theta$ & CMag & lp & $W p$ \\
\hline 80 & 150 & 0,8 & 52 & 0,57 & 0,010 & 2430 & 5440 & 1,5 & 5 & 25,50 & 0 & 3010,0 \\
\hline 80 & 150 & 0,8 & 48 & 0,60 & 0,033 & 2430 & 5760 & 1,5 & 5 & 30,13 & 4869,4 & 3167,7 \\
\hline 80 & 150 & 0,8 & 44 & 0,63 & 0,067 & 2430 & 6400 & 1,5 & 5 & 35,59 & 4882,0 & 3487,3 \\
\hline 80 & 150 & 0,8 & 42 & 0,65 & 0,070 & 2430 & 7040 & 1,5 & 5 & 38,69 & 4892,8 & 3728,5 \\
\hline 80 & 150 & 0,8 & 36 & 0,70 & 0,050 & 2430 & 6720 & 1,5 & 5 & 49,67 & 4909,9 & 4044,5 \\
\hline
\end{tabular}

incentivo adicional ao agente. 0 principal fica com um valor inferior à metade do acréscimo no lucro, devido ao custo de manutenção do agente.

A situação, sem terceirização, seria aquela em que a empresa manteria apenas seu padrão atual de manutenção, portanto lucro de $\$ 3.010,00$. Vê-se, na linha 2, que uma redução de 4 no número de peças não conformes conduz a um aumento no lucro de $\Delta W p=\$ 157,7$, o que satisfaz a restrição 13 . 0 lucro $(W p=\$ 3.167,70)$, obtido com a terceirização, representa o lucro sem terceirização mais a metade do aumento de ganho obtido com a redução de peças não conformes, subtraído o esforço adicional de custos para produzi-lo, porque o incentivo $(l p=4869,4)$ provoca a divisão desses lucros.

Alerta-se para o fato de que a diminuição de peças não conformes, que gerou o aumento do lucro, só foi possível porque os custos de se gerar essa redução para o agente, conforme Equação 17, não excederam o incentivo fornecido pelo principal. Portanto, sempre que o agente verificar que o seu custo de gerar uma melhoria nas variáveis $C, D$ e IQM for inferior ao incentivo que será recebido, ele irá agir no sentido de aumentar seu lucro, consequentemente o principal também terá aumento nos seus ganhos.

De posse dos resultados obtidos com o uso do modelo, verifica-se a importância da consideração dos incentivos baseados nas variáveis apontadas, conduzindo à cooperação entre o contratante $\mathrm{e}$ contratado, propósito fundamental da teoria principal-agente.

\section{Conclusão}

Diante da decisão de terceirizar as atividades de manutenção, e devido ao conflito de interesses entre contratante e contratado, a teoria principal-agente fornece subsídios para as empresas criarem contratos de incentivos que visem a cooperação. 0 modelo apresentado neste trabalho, fundamentado na teoria citada, modelou uma política de incentivos baseada não apenas no tempo total de manutenção, mas também na verificação da redução do número de peças defeituosas e na avaliação da capacidade de produção. Percebe-se a consistência do modelo ao se analisarem os resultados obtidos com o exemplo numérico utilizado, ressaltando-se, também, a necessidade de inclusão dessas variáveis nos modelos futuros. Confirmou-se que o modelo induz a uma cooperação entre agente e principal na obtenção de resultados positivos. Outra vantagem do modelo é a generalização, o que permite a aplicação das mais variadas funções de custo conforme a tecnologia do sistema. Abre-se, portanto, uma janela para aplicações mais aprofundadas, tais como aplicação em linhas de produção e não apenas em uma única máquina, a inclusão da incerteza para aperfeiçoamento das decisões e consideração de contratos de informação incompleta. 0 aumento do número de variáveis relevantes a serem consideradas na construção de modelos aumenta a complexidade do tratamento matemático, mas conduz a um aperfeiçoamento da consideração de aspectos que são fundamentais no processo de tomada de decisão na busca de melhoria dos resultados.

\section{Referências}

ALMEIDA, A. T. Multicriteria modelling of repair contract based on utility and ELECTRE 1 method with dependability and service quality criteria. Annals of Operations Research, v. 138, n. 1, 2005. p. 113-126. http://dx.doi.org/10.1007/ s10479-005-2448-z

ALMEIDA, A. T. Multicriteria decision making on maintenance: spares and contracts planning. European Journal of Operational Research, v. 129, n. 2, 2001. p. 235-241. http://dx.doi.org/10.1016/S0377-2217(00)00220-4

ALMEIDA, A. T.; SOUZA, F. M. C. Gestão da Manutenção: na direção da competitividade. Recife: Ed. Universitária, 2001.

AMADEO, E. J.; SCANDIUZZI, J. C.; PERO, V. Ajuste empresarial empregos e terceirização. Revista de Economia Política, v. 16 , n. 1, p. 21-36, 1996 
AMATO NETO, J. Reestruturação industrial, terceirização e redes de subcontratação. Revista de Administração de Empresas, v. 35, n. 2, p. 33-42,1995.

Ashgarizadeh, E.; Murthy, D. N. P. Service contracts: a stochastic model. Mathematical and Computer Modeling, v. 31 , n. 10 , p. 11-20, 2000. http://dx.doi.org/10.1016/ S0895-7177(00)00068-6

BEN-DAYA, M.; DUFFUAA, S. Maintenance and quality: the missing link. Journal of Quality in Maintenance Engineering, v. 1, n. 1, p. 20-26, 1995. http://dx.doi. org/10.1108/13552519510083110

FARIA, A. Terceirização: um desafio para o movimento sindical. In: MARTINS, H. H. T. S.; RAMALHO, J. R. (Orgs.).Terceirização: diversidade e negociação no mundo do trabalho. São Paulo: Hucitec, 1994.

GIOSA, L. A. Terceirização: uma abordagem estratégica. São Paulo: Pioneira, 1999.

HIGGINS, L. R.; MOBLEY, K. Maintenance Engineering Handbook. McGraw-Hill Professional, 2001.

JACKSON, C.; PASCUAL, R. Optimal maintenance service contract negotiation with aging equipment. European Journal of Operational Research, v. 189, n. 2, p. 387398, 2008. http://dx.doi.org/10.1016/j.ejor.2007.05.039

JENSEN, M. C.; MECKLING, W. H. Theory of the Firm: Managerial Behavior, Agency Costs and Ownership Structure. Journal of Financial Economics, v. 3, n. 4, p. 305-360, 1976. http://dx.doi.org/10.1016/0304-405X(76)90026-X

KUMAR, R.; MARKESET, T.; KUMAR, U. Maintenance of machinery: negotiating service contracts in businessto-business marketing. International Journal of Service Industry Management, v. 15, n. 3-4, p. 400-413, 2004. http://dx.doi.org/10.1108/09564230410552077

LAFFONT, J.-J.; MARTIMORT, D. The Theory of Incentives: The Principal-Agent Model. Princeton: University Press, 2001.

MARCHALEK, C. R. L.; REBELATO, M. G.; RODRIGUES, 1. C. Estudo sobre as diferenças de percepção dos fatores relacionais entre empresa e terceirizado. Produção, v. 17, n. 2, p. 286-301, 2007. http://dx.doi.org/10.1590/S010365132007000200006

MARCHI, M. M.; CARVALHO, S. V.; MORAIS, P. R. Um Modelo Estocástico para a Manutenção de um Equipamento Baseado na Inspeção das Peças Produzidas. Gestão e Produção, v. 8, n. 2, p. 115-127, 2001.

MAS-COLELL, A.; WHINSTON, M. D; GREEN, J. R. Microeconomic Theory. Oxford University Press, 1995.

MURTHY, D. N. P.; YEUNG, V. Modelling and analysis of maintenance service contracts. Mathematical and Computer Modelling, v. 22, n. 10-12, p. 219-225, 1995. http://dx.doi.org/10.1016/0895-7177(95)00199-C
MURTHY, D. N. P.; Ashgarizadeh, E. Optimal decision making in a maintenance service operation. European Journal of Operational Research, v. 116, p. 259-273, 1999. http:// dx.doi.org/10.1016/S0377-2217(98)90202-8

OKE, S. A.; AYOMOH, M. K. 0.; OYEDOKUN, 1. 0. An approach to measuring the quality of maintenance performance. IMA Journal Management Math, v. 18, p. 17-32, 2007. http://dx.doi.org/10.1093/imaman/dpi045

PALMER, R. D. Maintenance Planning and Scheduling Handbook. McGraw-Hill Professional, 1999.

PHAM, H. Handbook of Reliability Engineering. New Jersey: Springer, 2003. http://dx.doi.org/10.1007/b97414

SELLITTO, M. A. Formulação estratégica da manutenção industrial com base na confiabilidade dos equipamentos. Produção, v. 15, n. 1, p. 44-59, 2005 . http://dx.doi. org/10.1590/S0103-65132005000100005

STREMERSCH, S.; WUYTS, S.; FRAMBACH, R. The purchasing of full-service contracts: an exploratory study within the industrial maintenance market. Industrial Marketing Management, v. 30, n. 1, p. 1-12, 2001. http://dx.doi. org/10.1016/S0019-8501(99)00090-5

TARAKCl, H.; TANG, K.; TEYARACHAKUL, S. Learning effects on maintenance outsourcing. European Journal of Operational Research, v. 192, n. 1, p. 138-150, 2009. http://dx.doi.org/10.1016/j.ejor.2007.09.016

TAVARES, V. E. A gestão do risco na terceirização de atividades de manutenção mecânica: Um estudo de caso em uma indústria siderúrgica de grande porte. 2004. Dissertação (Mestrado)-Universidade Federal de Minas Gerais, 2004.

TSENG, F.-S. et al. Maintenance Outsourcing Contracts for New Technology Adoptions. IEEE Transactions on Engineering Management, v. 56, n. 2, p. 203-218, 2009. http://dx.doi. org/10.1109/TEM.2009.2013824

VALOIS, Ú.; ALMEIDA, A. T. Modelo de apoio à decisão multicritério para terceirização de atividades produtivas baseado no método SMARTS. Produção, v. 19, n. 2, p. 249-260, 2009. http://dx.doi.org/10.1590/ S0103-65132009000200003

VARIAN, H. R. Microeconomic Analysis. 3. ed. W.W. Norton e Co., 1992

WANG, W. A model for maintenance service contract design, negotiation and optimization. European Journal of Operational Research, v. 201, n. 1, p. 239-246, 2010. http://dx.doi.org/10.1016/j.ejor.2009.02.018

YEH, R. H., KAO, K. C.; CHANG, W. L. Optimal preventive maintenance policy for leased equipment using failure rate reduction. Computers and Industrial Engineering, v. 57, n. 1, p. 304-309, 2009.

\title{
Model contract for outsourcing of maintenance: a principal-agent approach
}

\begin{abstract}
The outsourcing is being used to reduce work on activities not related to production activities. Nowadays, the models of contracts of maintenance use the repair time and the cost of maintenance activities as main control indicator to estimate the political of incentives. The main objective of this paper is to introduce a model of incentives considering other variables that affect substantially the profit and that they are affected by the maintenance activities as the quality of products produced and the reduction of production capacity. The paper uses the Principal-Agent Theory to develop an incentive contract that leads the agent to execute activities that maximize the profit. It is used a numerical example in order to highlight the positive impact on company results, in addition to the generalization and adaptation of the model.
\end{abstract}

\section{Keywords}

Maintenance contract. Maintenance quality. Principal-agent theory. 